\title{
RNase activity of sialic acid-binding lectin from bullfrog eggs drives antitumor effect via the activation of p38 MAPK to caspase-3/7 signaling pathway in human breast cancer cells
}

\author{
YUKIKO KARIYA ${ }^{1}$, TAKEO TATSUTA ${ }^{1}$, SHIGEKI SUGAWARA ${ }^{1}$, YOSHINOBU KARIYA ${ }^{2}$, \\ KAZUO NITTA $^{1}$ and MASAHIRO HOSONO ${ }^{1}$ \\ ${ }^{1}$ Division of Cell Recognition Study, Institute of Molecular Biomembrane and Glycobiology, \\ Tohoku Pharmaceutical University, Sendai 981-8558; ${ }^{2}$ Department of Biochemistry, \\ Fukushima Medical University School of Medicine, Fukushima City, Fukushima 960-1295, Japan
}

Received March 18, 2016; Accepted July 20, 2016

DOI: 10.3892/ijo.2016.3656

\begin{abstract}
Sialic acid-binding lectin obtained from bullfrog eggs (SBL) induces cell death in cancer cells but not in normal cells. This antitumor effect is mediated through its ribonuclease (RNase) activity. However, the underlying molecular mechanisms remain unclear. We found that the p38 mitogenactivated protein kinase (MAPK) signaling pathway was activated when SBL induced cell death in three human breast cancer cell lines: SK-BR-3, MCF-7, and MDA-MB231. The suppression of p38 MAPK phosphorylation by a p38 MAPK inhibitor as well as short interference RNA knockdown of p38 MAPK expression significantly decreased cell death and increased the cell viability of SBL-treated MDA-MB231 cells. H103A, an SBL mutant lacking in RNase activity, showed decreased SBL-induced cell death compared with native SBL. However, the loss of RNase activity of SBL had no effect on its internalization into cells. The H103A mutant also displayed decreased phosphorylation of p38 MAPK. Moreover, SBL promoted caspase-3/7 activation followed by a cleavage of poly (ADP-ribose)-polymerase, whereas the SBL mutant, H103A, lost this ability. The SBL-induced caspase-3/7 activation was suppressed by the p38 MAPK inhibitor, SB203580,
\end{abstract}

Correspondence to: Professor Masahiro Hosono, Division of Cell Recognition Study, Institute of Molecular Biomembrane and Glycobiology, Tohoku Medical and Pharmaceutical University, 4-4-1 Komatsushima, Aoba-ku, Sendai 981-8558, Japan

E-mail:mhosono@tohoku-mpu.ac.jp

Abbreviations: CBB, Coomassie Brilliant Blue; DMSO, dimethyl sulfoxide; Gb3, globotriaosylceramide 3; IPTG, isopropyl 1-thio- $\beta$-Dgalactoside; mAb, monoclonal antibody; MAPK, mitogen-activated protein kinase; PARP, poly (ADP-ribose)-polymerase; PBS, phosphatebuffered saline; RNase, ribonuclease; SBL, sialic acid-binding lectin; SD, standard deviation; siRNA, short interference RNA

Key words: antitumor, cell death, p38 MAPK, RNase, sialic acidbinding lectin as well as pan-caspase inhibitor, zVAD-fmk. In the presence of zVAD-fmk, the SBL-induced cell death was decreased. In addition, the cell viability of SBL-treated MDA-MB231 cells recovered by zVAD-fmk treatment. Taken together, our results suggest that the RNase activity of SBL leads to breast cancer cell death through the activation of p38 MAPK followed by the activation of caspase-3/7.

\section{Introduction}

Sialic acid-binding lectin is a lectin from Rana catesbeiana oocytes (SBL). SBL preferentially binds to cancer cells rather than normal cells (1) because cancer cells often overexpress sialylated glycans on their surface, which is usually associated with poor prognosis (2). SBL shows an agglutination activity toward cancer cells by binding to the sialylated glycans on the surface of cancer cells (1).

SBL also exhibits a prominent antitumor effect on many types of cancer and tumor cells, such as breast, cervical, oral cancer, glioblastoma, and T-cell leukemia, but not normal cells, such as keratinocytes, fibroblasts, and lymphocytes (3-6). Moreover, the treatment of cancer cells with SBL ultimately leads to cell death (7). In mice with ascites tumor cells, the injection of SBL inhibits tumor growth and prolongs the life span, and sialidase protects cancer cells from SBL toxicity (8). Therefore, this selective antitumor effect of SBL is due to the sialylated glycans on the surface of tumors or cancer cells.

SBL is homologous with various members of the ribonuclease (RNase) A superfamily and is also known as RC-RNase $(9,10)$. The RNase A superfamily exhibits RNA-cleavage activity and has three catalytic amino acid residues. Therefore, SBL also has RNase activity and the conserved catalytic amino acid residues (His ${ }^{10}, \mathrm{Lys}^{35}$, and His ${ }^{103}$ ). Huang et al demonstrated that the three amino acid residues in the SBL molecule are required for inducing cancer cell death as well as RNase activity using recombinant SBL mutants with these amino acid residues replaced with alanine residues (11).

The internalization of SBL into cancer cells causes the degradation of ribosomal RNA, which leads to the inhibition of protein synthesis and, in turn, induces cell death $(8,12,13)$. 
SBL-induced cell death is accompanied by mitochondrial dysfunction (14), endoplasmic reticulum stress (15), autophagocytosis (16), and caspase activation $(3,5)$. Our previous studies showed that mitogen-activated protein kinases (MAPKs) were phosphorylated in two SBL-treated cell lines, human T-cell leukemia Jurkat cells and malignant mesothelioma NCI-H28 cells $(14,17)$. However, it remains unclear whether MAPK activation is related to SBL-induced cell death and how SBL activates MAPKs.

In this study, we found that SBL-induced cell death and activation of p38 MAPK signaling in human breast cancer cell lines. The analyses using p38 MAPK-specific inhibitors and short interference RNA (siRNA) showed that p38 MAPK activation and expression were associated with SBL-induced cell death. Furthermore, RNase activity of SBL was required for the observed SBL-induced cell death. SBL mutant lacking RNase activity indicated that such RNase activity of SBL was important for SBL-induced p38 MAPK activation and subsequent caspase-3/7 activation. Together, these data demonstrate that the RNA degradation by SBL triggers the SBL-induced p38 MAPK activation that leads to cell death mediated by caspase-3/7 activation.

\section{Materials and methods}

Antibodies and reagents. Mouse mAbs against p38 MAPK (no. 612168) and phospho-p38 MAPK (no. 612280) were obtained from BD Biosciences. A mouse mAb against $\beta$-actin (clone AC-74) was obtained from Sigma. A rabbit polyclonal antibody against PARP was obtained from Roche (no. 11835238001). A rabbit polyclonal SBL antibody was established in our laboratory. Alexa Fluor 488-conjugated goat anti-rabbit IgG (no. A11008) and Alexa Fluor 546-conjugated Phalloidin (no. A22283) were obtained from Invitrogen. Native SBL was isolated by sequential chromatography on Sephadex G-75, DEAE-cellulose, hydroxyapatite, and SP-Sepharose as described previously (1). Two types of p38 MAPK inhibitor (SB203580, no. 559389; and SB239063, no. 559404) and a JNK inhibitor (SP600125, no. 420119) were from Calbiochem. A pan-caspase inhibitor [zVAD-fmk, benzyloxycarbonylVal-Ala-Asp(OMe)-fluoromethylketone] was from MBL. Signal Silence p38 MAPK siRNA (no. 6564) and Signal Silence control siRNA (no. 6568) were from Cell Signaling Technology, Inc.

Cell culture. Human breast cancer cell lines, SK-BR-3, MCF-7, and MDA-MB231 were cultured in Dulbecco's modified Eagle's medium supplemented with $10 \%$ fetal bovine serum, penicillin, and streptomycin.

Cell viability. Cells were plated to each well of 96-well microtiter plates before treatment with test samples. After the treatment at $37^{\circ} \mathrm{C}$ for indicated time, WST-8 (Dojindo) was added to each well to detect viable cells. Color development was recorded at $450 \mathrm{~nm}$ using the PowerScan HT (DS Pharma Biomedical).

Expression vector encoding an SBL mutant. The full-length cDNA of SBL was prepared from a bullfrog liver. To prepare the expression vector of the SBL mutant lacking RNase activity, we replaced His ${ }^{103}$ of SBL with Ala by a PCR method using the SBL cDNA in pET11d with a pel $B$ sequence of the pET22b vector as a template. The cycle protocol for PCR was as follows: one cycle of $94^{\circ} \mathrm{C}$ for $2 \mathrm{~min} ; 25$ cycles of $94^{\circ} \mathrm{C}$ for $1 \mathrm{~min}, 51^{\circ} \mathrm{C}$ for $30 \mathrm{sec}$, and $68^{\circ} \mathrm{C}$ for $6 \mathrm{~min}$; and one cycle of $68^{\circ} \mathrm{C}$ for $4 \mathrm{~min}$. The primer set for H103A was $5^{\prime}$-GTAGCGT TTGCTGGAATAGGACGA-3' and 5'-GGGATATTGATT CTCACATTTTAC-3'. The cDNA sequence was verified by DNA sequencing.

Purification of recombinant SBL. E. coli BL21 (DE) pLysS was transformed with SBL cDNA expression vector and then selected with carbenicillin and chloramphenicol. The transformed cells were grown at $34^{\circ} \mathrm{C}$ in Terrific Broth medium containing $50 \mu \mathrm{g} / \mathrm{ml}$ carbenicillin and $34 \mu \mathrm{g} / \mathrm{ml}$ chloramphenicol until the A600 reached 0.6 before isopropyl 1-thio- $\beta$-D-galactoside (IPTG) induction. After IPTG induction at a final concentration of $0.4 \mathrm{mM}$ for 2 days, the culture supernatant was collected and then concentrated with $80 \%$ saturated ammonium sulfate. Pellet containing proteins were dissolved in $20 \mathrm{mM}$ HEPES buffer ( $\mathrm{pH} 7.0)$ and then dialyzed against the same buffer. The crude sample was loaded onto a DEAE-Sepharose 6B column (GE Healthcare), and the flow-through fraction was collected. The fraction was loaded on a Heparin-Sepharose 6 column (GE Healthcare) and eluted with $10 \mathrm{mM}$ Tris- $\mathrm{HCl}$ ( $\mathrm{pH} 7.5)$ containing $0.1-0.5 \mathrm{M} \mathrm{NaCl}$. The fractions containing H103A were loaded on a hydroxyapatite column after a threefold dilution with $10 \mathrm{mM}$ Tris- $\mathrm{HCl}$ (pH 7.5) and then the flow-through fraction was collected. The purified protein was resolved by SDS-PAGE and then stained with $\mathrm{CBB}$. The purified protein concentration was determined by DC protein assay (Bio-Rad Laboratories).

Knockdown of 38 MAPK by siRNA. Double-stranded siRNAs were transfected into MDA-MB231 cells using X-treme GENE siRNA Transfection Reagent (Roche) according to the instruction manual. After transfection for $24 \mathrm{~h}$, the cells were used for the following assays.

Analysis of RNA degradation. For analysis of intracellular RNA degradation, cells ( $2 \times 10^{4}$ cells) were grown in the medium containing SBL. After incubation for 24,48 , or $72 \mathrm{~h}$, total RNA of the cells was extracted with TRIzol reagent (Gibco BRL) according to the instruction manual. A volume of $2 \mu \mathrm{g}$ RNA was run on a $2 \%$ agarose gel containing formaldehyde, and the RNA bands were visualized by ethidium bromide.

Immunoblot analysis. Cells were washed twice with cold phosphate-buffered saline (PBS) and then lysed with lysis buffer (1\% Triton X-100, 20 mM Tris-HCl, pH 7.4, $150 \mathrm{mM}$ $\mathrm{NaCl}, 5 \mathrm{mM}$ EDTA) containing protease and phosphatase inhibitor cocktails (Roche). After incubation for $10 \mathrm{~min}$ on ice, the cell lysates were clarified by centrifugation at $20,400 \mathrm{~g}$ for $10 \mathrm{~min}$ at $4^{\circ} \mathrm{C}$. The resultant supernatant was used in the following experiments. The protein concentration was determined using a Protein Quantification Kit-Rapid (Dojindo). For immunoblot analysis, proteins resolved by SDS-PAGE were transferred to nitrocellulose membranes. The blots were blocked with $5 \%$ fat-free skim milk in TBS (20 mM Tris- $\mathrm{HCl}, 150 \mathrm{mM} \mathrm{NaCl}, \mathrm{pH}$ 7.4) for $1 \mathrm{~h}$. Then 

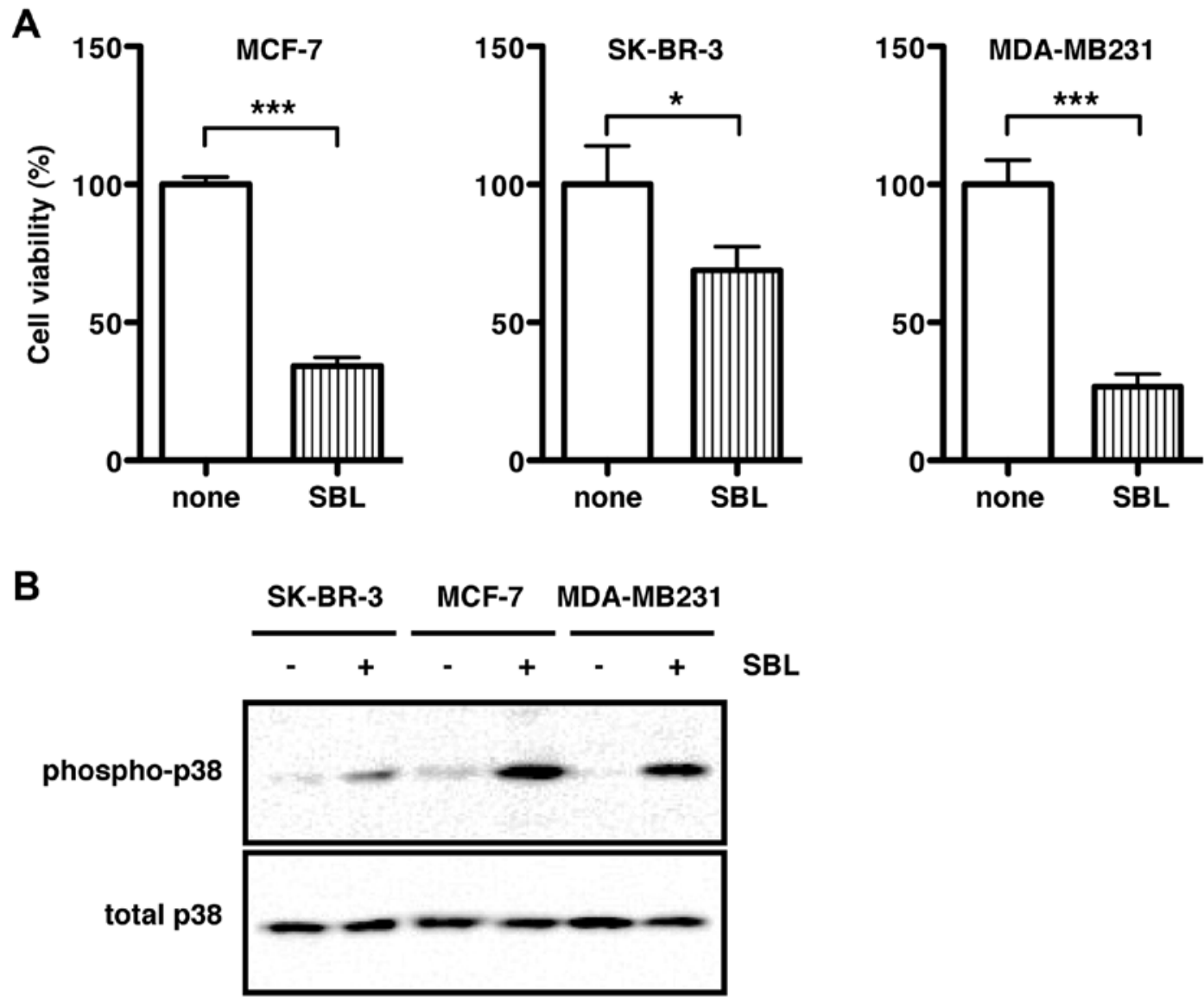

Figure 1. SBL induces cell death and activation of p38 MAPK in human breast cancer cell lines. (A) Cell viability of the indicated human breast cancer cell lines after treatment with $2 \mu \mathrm{M}$ SBL for $72 \mathrm{~h}$. The relative cell viability of cells with no treatment with SBL (none) was set at $100 \%$. Results are means \pm SD from three independent experiments conducted in triplicate for each experiment. ${ }^{*} \mathrm{P}<0.05$. ${ }^{* * *} \mathrm{P}<0.001$. (B) Cell lysates after treatment with or without $2 \mu \mathrm{M}$ SBL for $72 \mathrm{~h}$ were subjected to immunoblotting to detect phosphorylated p38 MAPK (upper panel) and total p38 MAPK (lower panel).

the membranes were probed with each specific antibody. Immunoreactive bands were detected using an ECL Western blotting detection reagent (GE Healthcare).

Immunofluorescence analysis. Cells were cultured in growth medium containing test samples. Cells were then fixed with 4\% paraformaldehyde in PBS for $10 \mathrm{~min}$ and permeabilized with $0.2 \%$ Triton X-100 in PBS for $10 \mathrm{~min}$. Cells were blocked with $1.2 \%$ BSA in PBS before staining with appropriate primary and secondary antibodies. After labeling with the indicated antibodies, labeled slides were analyzed using a fluorescent microscope, TIRFM (Olympus Corp.).

Caspase-3/7 activity. Caspase-3/7 activity was quantified using SensoLyte ${ }^{\circledR}$ Homogeneous AMC Caspase-3/7 Assay kit (AnaSpec Inc., no. 7118), according to the instruction manual.

Statistical analysis. Results are expressed as means \pm SD and are representative of at least two or three independent experiments conducted in triplicate for each experiment. Statistical comparisons between two groups were made using an unpaired Student's t-test and among groups using one-way ANOVA followed by a Tukey's multiple comparison test, with GraphPad Prism Version 5.0 software. A P-value of $<0.05$ was considered statistically significant.

\section{Results}

SBL induces cell death through 338 MAPK activation and expression in human breast cancer cell lines. SBL induces cell death in various types of human cancer cells (7). To examine the antitumor effect of SBL on breast cancer cells, we used one estrogen-positive cell line (MCF-7) and two estrogen-negative cell lines (SK-BR-3 and MDA-MB231) for a cell viability assay. After treatment with SBL for $72 \mathrm{~h}$, the cell viability of MCF-7, SK-BR-3, and MDA-MB231 was significantly reduced to $30.4,65.3$ and $25.5 \%$ of control levels (none, $100 \%$ ), respectively (Fig. 1A). As shown in our previous study using T-cell leukemia Jurkat cells and malignant mesothelioma NCI-H28 cells $(14,17)$, treatment with SBL markedly induced phosphorylation of p38 MAPK in all the three breast cancer cell lines, indicating that SBL activated the p38 MAPK signaling pathway (Fig. 1B).

The p38 MAPK inhibitor SB203580, sufficiently blocked SBL-induced phosphorylation of p38 MAPK (Fig. 2A, SB). In contrast, neither JNK inhibitor, SP600125 (Fig. 2A, SP), nor the pan-caspase inhibitor, VAD (Fig. 2A, VAD), had an effect on p38 MAPK phosphorylation. To investigate whether SBL-induced cell death was mediated by p38 MAPK activation, we tested the inhibitory effect of the p38 MAPK inhibitor SB203580, on SBL-induced cell death using MDA-MB231 
A

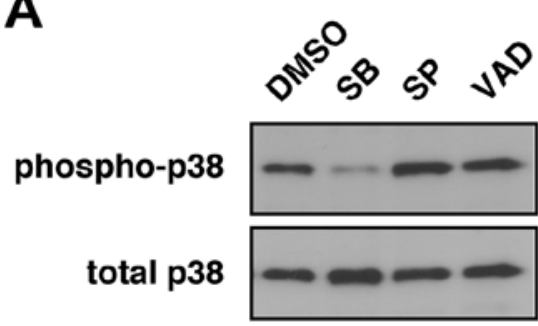

C

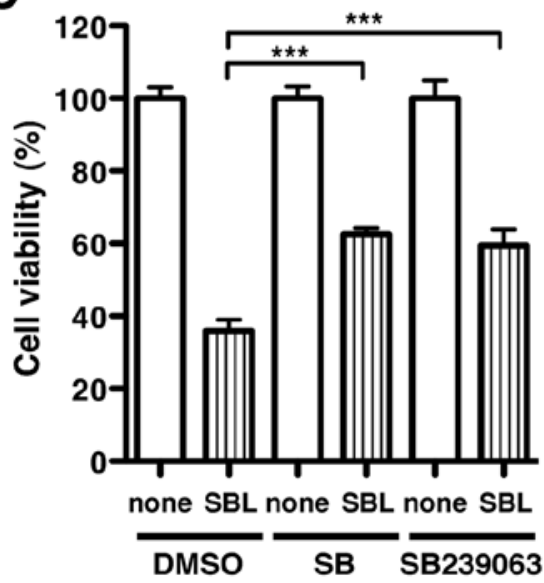

B

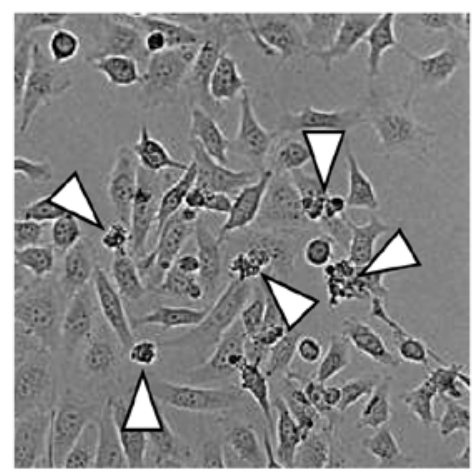

D

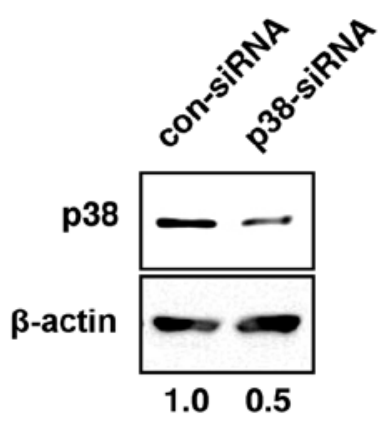

SB

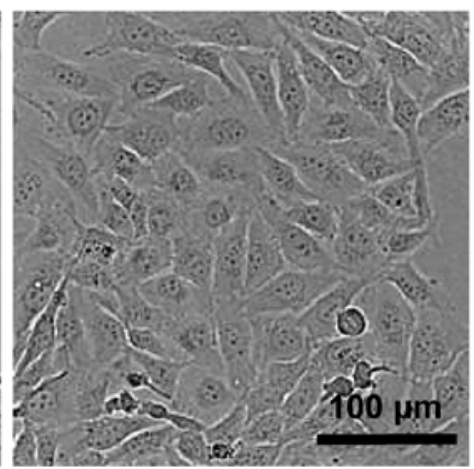

E

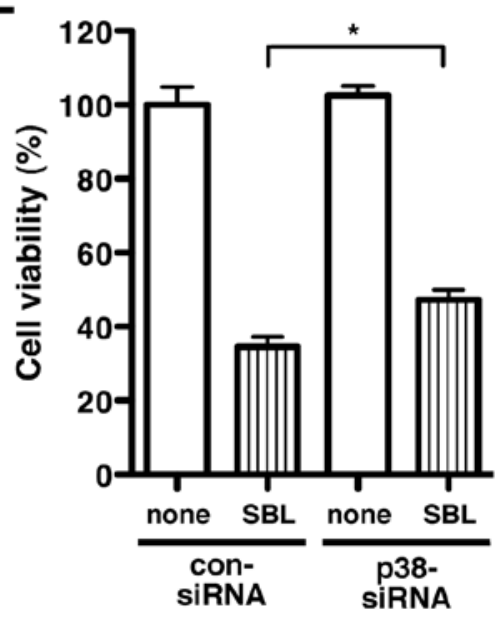

Figure 2. Inhibition of p38 MAPK activation and expression suppresses SBL-induced cell death in MDA-MB231 cells. (A) Immunoblot analysis of phosphorylated p38 MAPK (upper panel) and total p38 MAPK (lower panel) in MDA-MB231 cells treated with $2 \mu \mathrm{M}$ SBL in the presence of $1 \%$ (v/v) solvent (DMSO), $10 \mu \mathrm{M}$ SB203580 (SB), $10 \mu \mathrm{M}$ SP600125 (SP), or $100 \mu \mathrm{M}$ zVAD-fmk (VAD) for $72 \mathrm{~h}$. (B) Cell morphology of MDA-MB231 cells after treatment with $2 \mu \mathrm{M}$ SBL for $72 \mathrm{~h}$ in the presence of DMSO or $10 \mu \mathrm{M} \mathrm{SB}$. Arrowheads indicate dying or dead cells. (C) Cell viability after treatment with or without SBL for $72 \mathrm{~h}$ in the presence of DMSO, $10 \mu \mathrm{M}$ SB or $10 \mu \mathrm{M}$ SB239063. The relative cell viability of cells with no treatment with SBL (none) in each sample, was set at $100 \%$. ${ }^{* * * *} \mathrm{P}<0.001$. (D) Cell lysates treated with control siRNA (con-siRNA) or p38 MAPK-specific siRNA (p38-siRNA) were subjected to immunoblotting to detect endogenous p38 MAPK and $\beta$-actin. The numbers at the bottom indicate the ratio of the intensity of p38 MAPK to the intensity of $\beta$-actin in each sample, which was 1.0 for con-siRNA-treated cells. (E) The viability of cells treated with con-siRNA or p38-siRNA. The relative cell viability of cells with no treatment with SBL for $48 \mathrm{~h}$ (none) in each sample was set at $100 \%$. Results are the means \pm SD for three independent experiments conducted in triplicate. $\mathrm{P}<0.05$.

cells. When treated with SBL, some MDA-MB231 cells shrank and were dying or dead [Fig. 2B, dimethyl sulfoxide (DMSO)]. The SBL-induced cell death was markedly inhibited by the addition of SB203580 (Fig. 2B, SB). The p38 MAPK inhibitors, SB203580 and SB239063 improved the cell viability in SBL-treated cells from 36\% (Fig. 2C, DMSO/SBL) to $63 \%$ (Fig. 2C, SB/SBL) and 59\% (Fig. 2C, SB239063/SBL), respectively.

To further investigate the relationship between SBL-induced cell death and the p38 MAPK signaling pathway, we performed a cell viability assay using a specific siRNA to p38 MAPK (p38-siRNA). The expression of the endogenous p38 MAPK molecule was assessed after $96 \mathrm{~h}$ transfection of p38-siRNA or scrambled control siRNA (con-siRNA). When MDA-MB231 cells were treated with p38-siRNA, the expression level of the p38 MAPK molecule was suppressed to a half of that treated with con-siRNA (Fig. 2D). After treating with siRNA for $24 \mathrm{~h}$, cells were further treated with SBL for $48 \mathrm{~h}$. The cell viability in the presence of SBL was partially rescued by the knockdown of p38 MAPK molecule (Fig. 2E). These results suggest that SBL induces cell death of the cancer cells through activation of $\mathrm{p} 38 \mathrm{MAPK}$.

RNase activity is required for SBL-induced cell death. SBL exhibits RNase activity. When cells were treated with SBL, 28S and 18S ribosomal RNA bands decreased in a time-dependent manner, and some degraded RNA bands were detected via formaldehyde-agarose gel electrophoresis (Fig. 3A). RNase activity has been shown to be necessary for SBL to degrade RNA, as well as to cause cell death of cancer cells (11). However, it remains unclear how the SBL-induced RNA degradation leads to cell death. To clarify the relationship between RNase activity and SBL-induced cell death, we prepared a recombinant SBL mutant that lacks RNase activity by replacing $\mathrm{His}^{103}$ (important for its RNase and cell deathinduced activities) with Ala (Fig. 3B, H103A). Escherichia coli BL21 (DE) pLysS was transformed with H103A cDNA expression vector, and then the recombinant protein was purified from the culture medium of H103A-expressing BL21 (DE) pLysS using several columns. 


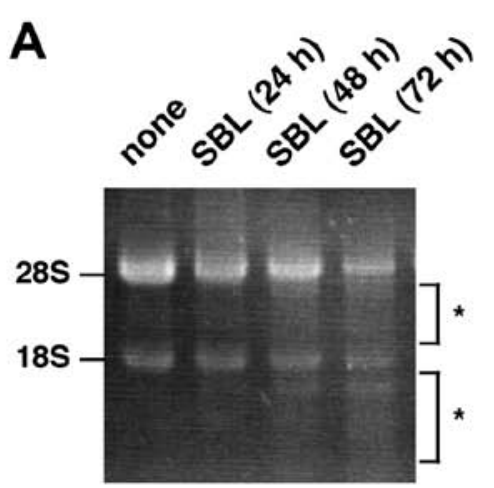

D

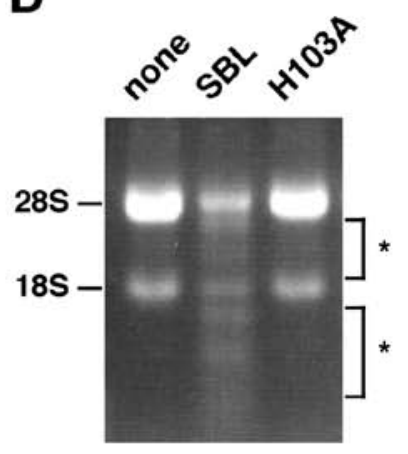

E

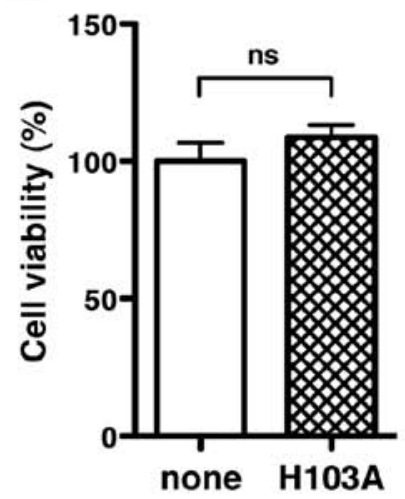

111

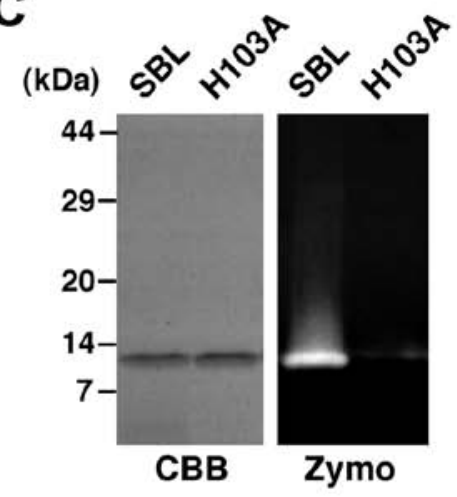

$\mathbf{F}$
103

SBL

H103A

95

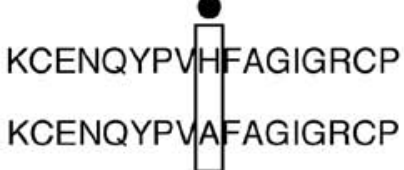

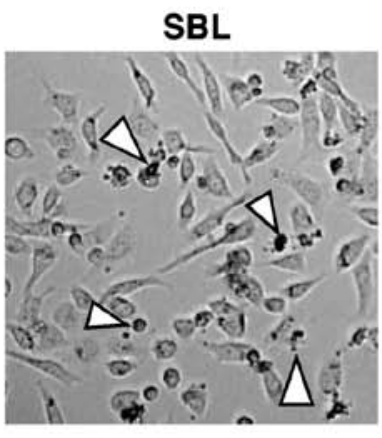

H103A

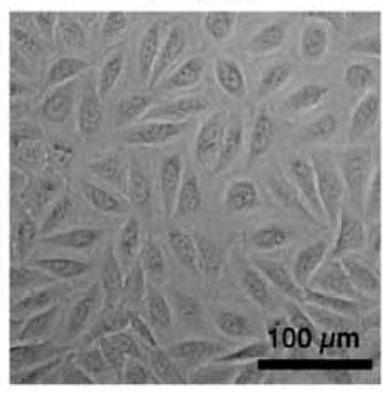

G

none

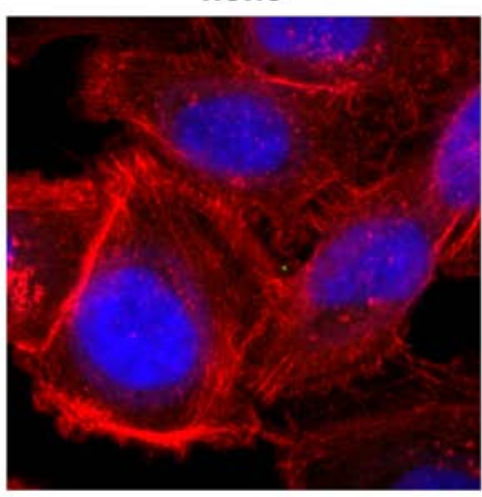

SBL
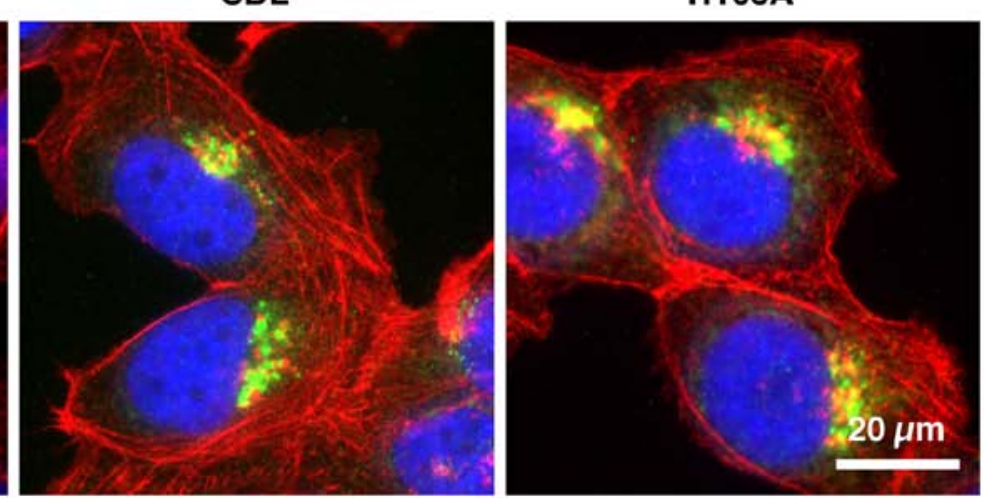

Figure 3. RNase activity of SBL is required for SBL-induced cell death. (A) RNA was extracted from MDA-MB231 cells treated with or without SBL for the indicated times and then analyzed on a formaldehyde-agarose gel. Asterisks indicate degraded RNA. None, no SBL treatment for $72 \mathrm{~h}$. SBL, $2 \mu \mathrm{M}$ SBL treatment. (B) For preparing an SBL mutant lacking RNase activity (H103A), the histidine residue at position 103 essential for its catalytic activity was replaced with an alanine residue (black dot). Numbers indicate the number of amino acid residues. (C) Purified proteins were run on 15\% SDS-PAGE gel under reducing conditions and stained with CBB (left panel). RNase activity of the purified proteins was analyzed by RNA substrate zymography under non-reducing condi-

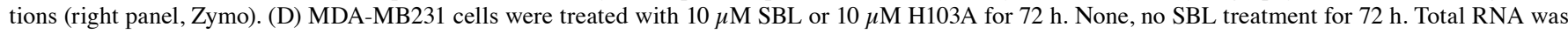
analyzed by formaldehyde-agarose gel electrophoresis. Asterisks indicate degraded RNA. (E) Cell viability after treatment with $10 \mu \mathrm{M} \mathrm{H} 103 \mathrm{~A}$ for $72 \mathrm{~h}$. The relative cell viability of cells with no treatment with SBL (none) was set at $100 \%$. Results are the means \pm SD for three independent experiments conducted in triplicate. ns, not significant. (F) Cell morphology of MDA-MB231 cells after treatment with $2 \mu \mathrm{M}$ SBL or $10 \mu \mathrm{M} \mathrm{H103A}$ for $72 \mathrm{~h}$. Arrowheads indicate dying or dead cells. (G) MDA-MB231 cells treated with $4 \mu \mathrm{M} \mathrm{SBL}$ (middle), $4 \mu \mathrm{M} \mathrm{H103A}$ (right), or without SBL (left, none) for $6 \mathrm{~h}$ were fixed with $4 \%$ paraformaldehyde, permeabilized with $1 \%$ Triton X-100/PBS, and then stained with anti-SBL antibody (green), phalloidin (red), and Hoechst 33342 (blue).

The quality of the purified H103A protein was assessed by Coomassie Brilliant Blue (CBB) staining, and its molecular mass was estimated to be $13 \mathrm{kDa}$, which was the same as that of native SBL protein (Fig. 3C, CBB). In a zymographic assay using an RNA-containing gel, native SBL exhibited RNase activity, but H103A did not (Fig. 3C, Zymo). The cellular RNA from the treated cells with native SBL was considerably degraded at $72 \mathrm{~h}$ while H103A and no SBL treatment did not induce the cellular RNA degradation (Fig. 3D). Furthermore, H103A had no effect on cell viability (Fig. 3E). In agreement with the result of cell viability, the H103A mutant did not initiate a cell death phenotype as observed with the native SBL (Fig. 3F). These results indicate that RNase activity is closely related to SBL-induced cell death.

The internalization of SBL into cells is required for SBL to degrade cellular RNA (12). To exclude the possibility 

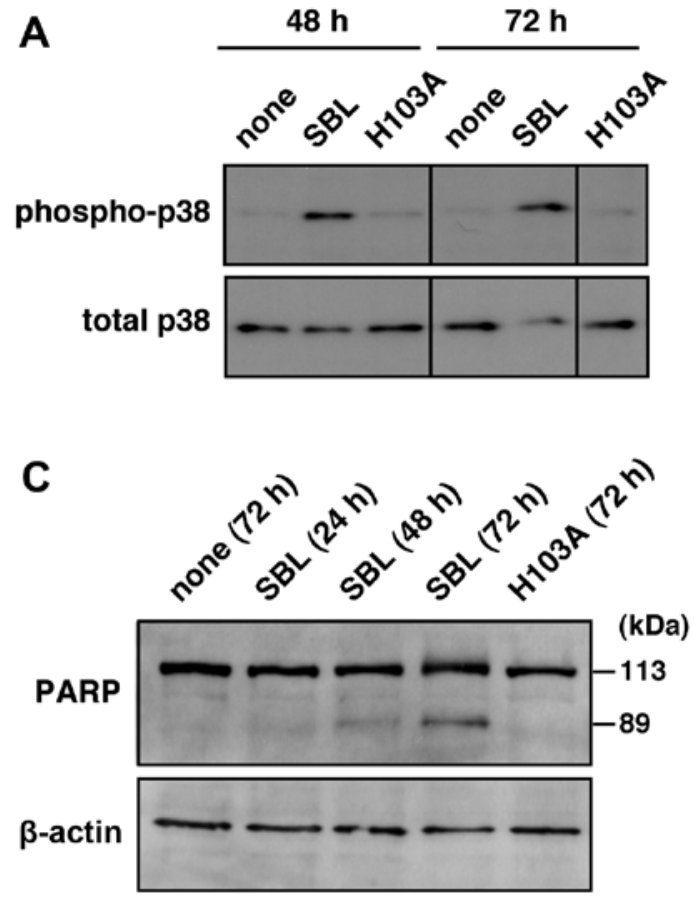

B

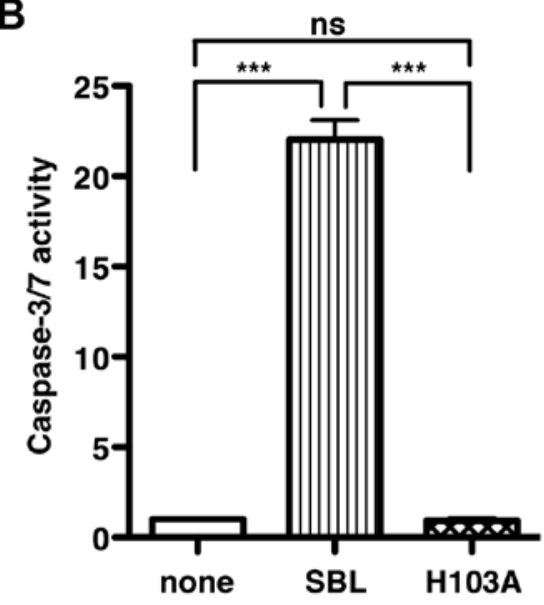

Figure 4. RNase activity of SBL is essential for SBL-induced p38 MAPK and caspase-3/7 activation. (A) Cell lysates of MDA-MB231 treated with $2 \mu \mathrm{M}$ SBL or $2 \mu \mathrm{M} \mathrm{H103A}$ for the indicated times were subjected to immunoblotting to detect phosphorylated p38 MAPK (upper panel) and total p38 MAPK (lower panel). Thin vertical lines indicate the removal of an intervening lane for presentation purposes. (B) Caspase-3/7 activity in MDA-MB231 cells treated with $2 \mu \mathrm{M}$ SBL or $10 \mu \mathrm{M} \mathrm{H103A}$ for $72 \mathrm{~h}$ was assessed using SensoLyte Homogeneous AMC Caspase-3/7 assay kit. Results are the means \pm SD for three independent experiments conducted in triplicate. ${ }^{* * *} \mathrm{P}<0.001$. ns, not significant. None, no SBL treatment. (C) Cell lysates of MDA-MB231 cells treated with $2 \mu \mathrm{M}$ SBL or $10 \mu \mathrm{M} \mathrm{H103A}$ for the indicated times were subjected to immunoblotting to detect PARP and $\beta$-actin. The smaller size of PARP, approximately $89 \mathrm{kDa}$, is a cleaved form of PARP, which is correlated to caspase-3/7 activation.

that $\mathrm{H} 103 \mathrm{~A}$ is not capable of cellular internalization, the intracellular localization of H103A was observed. H103A and native SBL were localized to the perinuclear region in MDA-MB231 cells at $6 \mathrm{~h}$ (Fig. 3G), indicating that H103A is internalized.

As shown in Figs. 1 and 2, p38 MAPK activation was associated with SBL-induced cell death. Therefore, it is likely that the RNase activity of SBL may be of relevance to p38 MAPK activation. We then examined the activation of p38 MAPK after treatment with native SBL and the H103A mutant. Native SBL increased the phosphorylation of p38 MAPK both at 48 and $72 \mathrm{~h}$. In contrast, H103A, as well as the untreated controls, did not promote the phosphorylation of p38 MAPK even at $72 \mathrm{~h}$ (Fig. 4A). These results revealed that the RNase activity of SBL is required for SBL-induced p38 MAPK activation.

RNA degradation by SBL leads to activation of caspase-3/7. Although SBL has been reported to activate caspase-3/7 $(3,5,18)$, known as the major executioner caspases, the molecular mechanism remains unclear. Indeed, the caspase-3/7 activity in SBL-treated MDA-MB231 cells was 22-fold higher than that in the untreated cells (Fig. 4B, none vs. SBL). This upregulated caspase-3/7 activity by SBL was completely ablated in the H103A mutant (Fig. 4B, H103A).

Active caspase-3/7 cleaves a full-length poly (ADP-ribose)polymerase (PARP; $113 \mathrm{kDa}$ ) into two fragments: a) $89 \mathrm{kDa}$ and b) $24 \mathrm{kDa}$. Therefore, we also examined SBL-induced caspase-3/7 activation by checking the fragments of PARP. The immunoblot analysis using an anti-PARP antibody showed that SBL treatment caused an increase in the $89-\mathrm{kDa}$ PARP fragment in a time-dependent manner, whereas only a 113-kDa PARP band was detected when H103A was treated for $72 \mathrm{~h}$ (Fig. 4C). These results indicate that RNase activity of $\mathrm{SBL}$ is required for SBL-induced caspase-3/7 activation.

Activation of p38 MAPK signaling induced by SBL leads to activation of caspase-3/7. Since RNA degradation by SBL remarkably induced p38 MAPK and caspase-3/7 activation, we speculated that SBL-induced p38 MAPK activation might be related to caspase-3/7 activation. To test this hypothesis, we examined caspase-3/7 activity after treatment with SBL in the presence of a p38 MAPK inhibitor. Caspase-3/7 activity after co-treatment of the p38 MAPK inhibitor, SB203580, with SBL was suppressed by $41.8 \%$ compared with that after co-treatment with the solvent, DMSO and SBL (Fig. 5A, SB). These results indicate that the activation of p38 MAPK by SBL leads to caspase-3/7 activation.

A pan-caspase inhibitor, zVAD-fmk, completely inhibited the caspase-3/7 activation induced by SBL (Fig. 5A, VAD). Furthermore, zVAD-fmk treatment inhibited the cleavage of PARP increased by SBL (Fig. 5B). Using zVAD-fmk, we then studied how active caspase-3/7 contributes to SBL-induced cell death. In the presence of zVAD-fmk, SBL-induced cell death decreased (Fig. 5C). In addition, zVAD-fmk increased the cell viability of SBL-treated MDA-MB231 cells compared with the control (Fig. 5D). These results suggest that SBL induces cell death of cancer cells through p38 MAPK-mediated activation of caspase-3/7. 


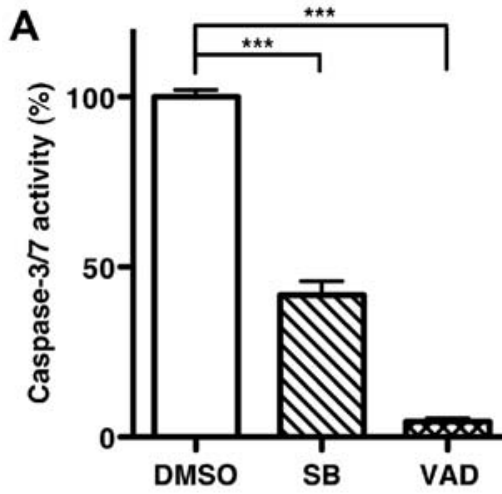

C

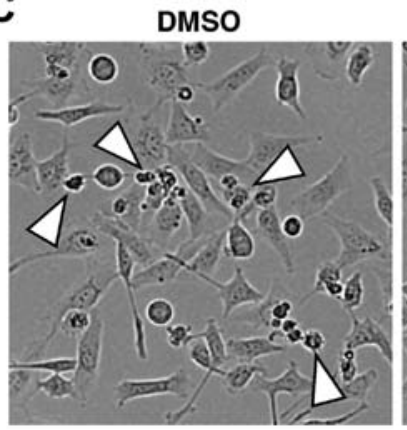

B

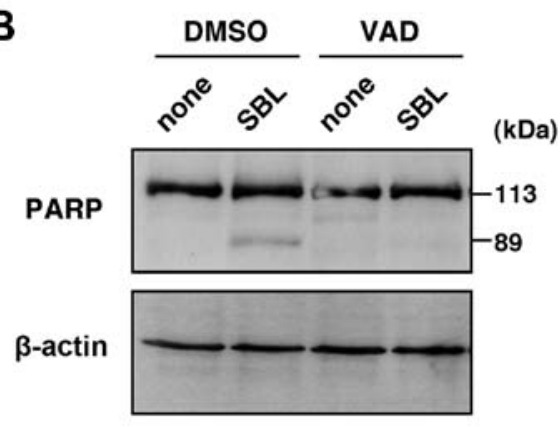

VAD

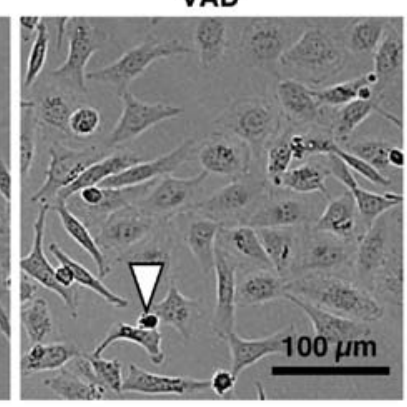

D

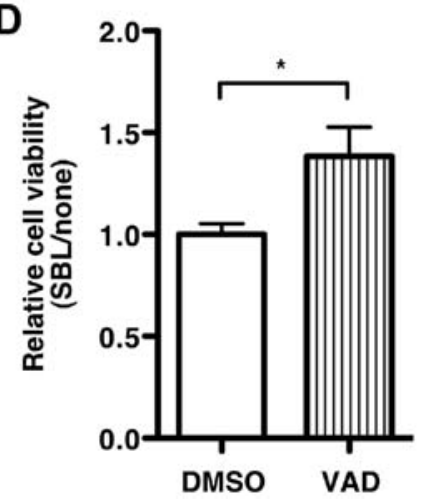

Figure 5. SBL induces caspase-3/7 activation. (A) Caspase-3/7 activity in MDA-MB231 cells treated with or without $2 \mu$ M SBL in the presence of solvent DMSO, $10 \mu \mathrm{M} \mathrm{p} 38$ MAPK inhibitor SB203580 (SB) or $100 \mu \mathrm{M}$ pan-caspase inhibitor zVAD-fmk (VAD) was assessed using SensoLyte Homogeneous AMC Caspase-3/7 assay kit. Each bar indicates the ratio of caspase-3/7 activity of the cells with SBL treatment to without SBL treatment. Results are means \pm SD for two independent experiments conducted in triplicate. ${ }^{* * *} \mathrm{P}<0.001$. (B) Cell lysates of MDA-MB231 cells treated with or without $2 \mu \mathrm{M}$ SBL in the presence of DMSO or $100 \mu \mathrm{M}$ zVAD-fmk (VAD) for $72 \mathrm{~h}$ were subjected to immunoblotting to detect PARP and $\beta$-actin. The smaller size of PARP, approximately $89 \mathrm{kDa}$, is a cleaved form of PARP, which is correlated to caspase-3/7 activation. (C) Cell morphology of MDA-MB231 cells after treatment with SBL for $72 \mathrm{~h}$ in the presence of solvent DMSO or $100 \mu \mathrm{M}$ zVAD-fmk (VAD). Arrowheads indicate dying or dead cells. (D) MDA-MB231 cells were treated with or without $2 \mu \mathrm{M}$ SBL in the presence of DMSO or $100 \mu \mathrm{M}$ zVAD-fmk (VAD) for $72 \mathrm{~h}$. Each bar indicates the ratio of cell viability with or without SBL treatment. ${ }^{*} \mathrm{P}<0.05$.

\section{Discussion}

Our previous study showed that SBL induced MAPK phosphorylation. However, it remains unclear whether the MAPK activation is related to SBL-induced cell death and RNase activity of SBL. In this study, we demonstrate that SBL induces the phosphorylation of p38 MAPK, due to RNA degradation by the RNase activity of SBL. We also find that SBL-induced p38 MAPK activation leads to caspase-3/7 activation and subsequent cell death.

In this study, the p38 MAPK inhibitor, SB inhibited caspase-3/7 activity (Fig. 5A) but caspase-3/7 inhibitor, VAD did not show any inhibitory effect on $\mathrm{p} 38$ MAPK phosphorylation (Fig. 2A). This suggests that p38 MAPK is an upstream regulator of caspase-3/7. However, SB did not completely inhibit caspase-3/7 activity (Fig. 5A). These results indicate that caspase-3/7 may be also activated by a signaling pathway other than p38 MAPK. Iordanov et al showed that both JNK and p38 MAPK are activated by onconase, which is an RNase isolated from Rana pipiens oocytes, and that JNK is related to the cleavage of PARP and onconase-induced cell death (19). Since SBL also activates JNK (14,17), JNK activation may also contribute to capase-3/7 activation resulting in SBL-induced cell death.

Although the p38 MAPK inhibitor, SB significantly inhibited SBL-induced cell death (Fig. 2C), approximately two-fifths of SB-treated cells still induced SBL-dependent cell death. Given that H103A efficiently abrogated SBL-induced cell death (Fig. 3E), there could also be p38 MAPK-independent signaling pathways for SBL-induced cell death. Inhibition of protein synthesis is most likely related to this pathway because RNA damage leads to an inhibition of protein synthesis in addition to p38 MAPK activation, and inhibition of protein synthesis was observed in SBL-treated cells (8).

SBL-induced cell death and p38 MAPK phosphorylation in SK-BR-3 cells were much weaker than those of MCF-7 and MDA-MB231 cells. SBL requires at least three steps to induce cell death: binding to cell surface via sialic acid, entering into cells, followed by the activation of cell death-related signaling. From the immunoblot analysis of cell lysate of SBL-treated cells, the amount of SBL entered into the cells was lower in SK-BR-3 cells than in MDA-MB231 and MCF-7 cells, suggesting that SBL inefficiently enters into SK-BR-3 cells compared to MDA-MB231 and MCF-7 cells (data not shown). Therefore, the weak reactivity against the SBL-induced cell death and p38 MAPK phosphorylation in SK-BR-3 cells might be due to the problem in the low binding of SBL to cell surface of SK-BR-3 cells. Further studies such as sialylation state and unidentified receptors in the cells are required to address the question.

Shiga toxin, also known as verotoxin 1 , acts as a protein synthesis inhibitor within target cells. Shiga toxin cleaves a 
specific adenine from $28 \mathrm{~S}$ rRNA by its RNA $N$-glycosidase activity, thereby inhibiting protein synthesis $(20,21)$. Moreover, Shiga toxin activates p38 MAPK or JNK $(22,23)$. The ability of Shiga toxin to activate p38 MAPK and JNK was lost by heat denaturation or substituted mutation in the active site, indicating that the p38 MAPK and JNK activation by Shiga toxin depend on its RNA $N$-glycosidase activity (22). Since an inhibitor of the p38 MAPK was demonstrated to block the cytotoxicity of Shiga toxin (23), Shiga toxin induces cell death via the p38 MAPK signaling pathway. Thus, the molecular mechanisms of Shiga toxin-induced cell death are mediated in large part by the ribotoxic stress response and are very similar to that of SBL-induced cell death presented in this study. The B subunit of Shiga toxin binds to globotriaosylceramide 3 (Gb3) on the host cells, followed by the RNA $\mathrm{N}$-glycosidase activity containing A subunit internalizing into cells. Although the mechanism of the internalization of SBL into cells is still unknown, sialoglycoproteins may work as a receptor for SBL-internalization, like Gb3 for the B subunit of Shiga toxin.

Onconase strongly induces cell death depending on its RNase activity $(24,25)$ by leading to the suppression of cell cycle progression, followed by apoptosis (26). Onconase has also been shown to have effective anticancer activity in animal models (27). Moreover, onconase has been studied in advanced phase IIIb clinical trials against malignant mesothelioma (28). Similar to onconase, SBL also shows high toxicity against some cancer cells and has antitumor effects in mouse models $(3,8)$. Moreover, SBL does not kill primary or normal cells because SBL recognizes and selectively binds to sialoglycoproteins on tumor and cancer cells $(1,7,18)$. Therefore, SBL may work as a potential antitumor drug as well as onconase.

In conclusion, our findings demonstrate an important role for RNase activity-dependent p38 MAPK activation and the subsequent caspase-3/7 activation in SBL-induced cancer cell death. Further studies regarding the molecular mechanism of SBL-induced cancer cell death may be helpful to the development of anticancer therapies in the future.

\section{Acknowledgements}

We appreciate Ms. Yuki Miura and Dr Kohta Takahashi (Tohoku Pharmaceutical University, Sendai) for their assistance. We also appreciate Dr Takashi Sugino (Shizuoka Cancer Center, Shizuoka) for his kind gift of breast cancer cell lines. This study was supported by the grant-in-aid for the 'Academic Frontier' Project (2006-2011) for Private Universities from the Ministry of Education, Culture, Sports, Science and Technology of Japan; and a Grant-in-Aid for Young Scientists (B) [grant no. 20790073 (to Yukiko Kariya)].

\section{References}

1. Nitta K, Takayanagi G, Kawauchi H and Hakomori S: Isolation and characterization of Rana catesbeiana lectin and demonstration of the lectin-binding glycoprotein of rodent and human tumor cell membranes. Cancer Res 47: 4877-4883, 1987.

2. Dall'Olio F and Chiricolo M: Sialyltransferases in cancer. Glycoconj J 18: 841-850, 2001.

3. Hu CC, Tang $\mathrm{CH}$ and Wang JJ: Caspase activation in response to cytotoxic Rana catesbeiana ribonuclease in MCF-7 cells. FEBS Lett 503: 65-68, 2001.
4. Liao YD, Huang HC, Chan HJ and Kuo SJ: Large-scale preparation of a ribonuclease from Rana catesbeiana (bullfrog) oocytes and characterization of its specific cytotoxic activity against tumor cells. Protein Expr Purif 7: 194-202, 1996.

5. Chen JN, Yiang GT, Lin YF, Chou PL, Wu TK, Chang WJ Chen $\mathrm{C}$ and $\mathrm{Yu}$ YL: Rana catesbeiana ribonuclease induces cell apoptosis via the caspase-9/-3 signaling pathway in human glioblastoma DBTRG, GBM8901 and GBM8401 cell lines. Oncol Lett 9: 2471-2476, 2015.

6. Ogawa Y, Sugawara S, Tatsuta T, Hosono M, Nitta K, Fujii Y, Kobayashi H, Fujimura T, Taka H, Koide Y, et al: Sialylglycoconjugates in cholesterol-rich microdomains of P388 cells are the triggers for apoptosis induced by Rana catesbeiana oocyte ribonuclease. Glycoconj J 31: 171-184, 2014.

7. Tatsuta T, Sugawara S, Takahashi K, Ogawa Y, Hosono M and Nitta K: Cancer-selective induction of apoptosis by leczyme. Front Oncol 4: 139, 2014.

8. Nitta K, Ozaki K, Ishikawa M, Furusawa S, Hosono M, Kawauchi H, Sasaki K, Takayanagi Y, Tsuiki S and Hakomori S: Inhibition of cell proliferation by Rana catesbeiana and Rana japonica lectins belonging to the ribonuclease superfamily. Cancer Res 54: 920-927, 1994.

9. Liao YD: A pyrimidine-guanine sequence-specific ribonuclease from Rana catesbeiana (bullfrog) oocytes. Nucleic Acids Res 20: 1371-1377, 1992.

10. Titani K, Takio K, Kuwada M, Nitta K, Sakakibara F, Kawauchi H, Takayanagi $\mathrm{G}$ and Hakomori S: Amino acid sequence of sialic acid binding lectin from frog (Rana catesbeiana) eggs. Biochemistry 26: 2189-2194, 1987.

11. Huang HC, Wang SC, Leu YJ, Lu SC and Liao YD: The Rana catesbeiana rcr gene encoding a cytotoxic ribonuclease. Tissue distribution, cloning, purification, cytotoxicity, and active residues for RNase activity. J Biol Chem 273: 6395-6401, 1998

12. Nitta K, Ozaki K, Tsukamoto Y, Furusawa S, Ohkubo Y, Takimoto H, Murata R, Hosono M, Hikichi N, Sasaki K, et al: Characterization of a Rana catesbeiana lectin-resistant mutant of leukemia P388 cells. Cancer Res 54: 928-934, 1994.

13. Nitta K, Ozaki K, Tsukamoto Y, Hosono M, Ogawakonno Y, Kawauchi H, Takayanagi Y, Tsuiki S and Hakomori S: Catalytic lectin (leczyme) from bullfrog (Rana catesbeiana) eggs. Int J Oncol 9: 19-23, 1996.

14. Tatsuta T, Hosono M, Sugawara S, Kariya Y, Ogawa Y, Hakomori S and Nitta K: Sialic acid-binding lectin (leczyme) induces caspase-dependent apoptosis-mediated mitochondrial perturbation in Jurkat cells. Int J Oncol 43: 1402-1412, 2013.

15. Tatsuta T, Hosono M, Miura Y, Sugawara S, Kariya Y, Hakomori S and Nitta K: Involvement of ER stress in apoptosis induced by sialic acid-binding lectin (leczyme) from bullfrog eggs. Int J Oncol 43: 1799-1808, 2013.

16. Yiang GT, Yu YL, Chou PL, Tsai HF, Chen LA, Chen YH, Su KJ, Wang JJ, Bau DT and Wei CW: The cytotoxic protein can induce autophagocytosis in addition to apoptosis in MCF-7 human breast cancer cells. In Vivo 26: 403-409, 2012.

17. Tatsuta T, Hosono M, Takahashi K, Omoto T, Kariya Y, Sugawara S, Hakomori S and Nitta K: Sialic acid-binding lectin (leczyme) induces apoptosis to malignant mesothelioma and exerts synergistic antitumor effects with TRAIL. Int J Oncol 44: 377-384, 2014.

18. Tang $\mathrm{CH}, \mathrm{Hu} \mathrm{CC}$, Wei $\mathrm{CW}$ and Wang JJ: Synergism of Rana catesbeiana ribonuclease and IFN-gamma triggers distinct death machineries in different human cancer cells. FEBS Lett 579: 265-270, 2005.

19. Iordanov MS, Wong J, Newton DL, Rybak SM, Bright RK, Flavell RA, Davis RJ and Magun BE: Differential requirement for the stress-activated protein kinase/c-Jun $\mathrm{NH}(2)$-terminal kinase in RNAdamage-induced apoptosis in primary and in immortalized fibroblasts. Mol Cell Biol Res Commun 4: 122-128, 2000.

20. Endo Y, Tsurugi K, Yutsudo T, Takeda Y, Ogasawara T and Igarashi K: Site of action of a Vero toxin (VT2) from Escherichia coli $\mathrm{O} 157: \mathrm{H} 7$ and of Shiga toxin on eukaryotic ribosomes. RNA $N$-glycosidase activity of the toxins. Eur J Biochem 171: 45-50, 1988.

21. Saxena SK, O'Brien AD and Ackerman EJ: Shiga toxin, Shiga-like toxin II variant, and ricin are all single-site RNA $\mathrm{N}$-glycosidases of $28 \mathrm{~S}$ RNA when microinjected into Xenopus oocytes. J Biol Chem 264: 596-601, 1989.

22. Smith WE, Kane AV, Campbell ST, Acheson DW, Cochran BH and Thorpe CM: Shiga toxin 1 triggers a ribotoxic stress response leading to $\mathrm{p} 38$ and JNK activation and induction of apoptosis in intestinal epithelial cells. Infect Immun 71: 1497-1504, 2003. 
23. Ikeda M, Gunji Y, Yamasaki S and Takeda Y: Shiga toxin activates p38 MAP kinase through cellular $\mathrm{Ca}(2+)$ increase in Vero cells. FEBS Lett 485: 94-98, 2000.

24. Wu Y, Mikulski SM, Ardelt W, Rybak SM and Youle RJ: A cytotoxic ribonuclease. Study of the mechanism of onconase cytotoxicity. J Biol Chem 268: 10686-10693, 1993.

25. Lee JE and Raines RT: Contribution of active-site residues to the function of onconase, a ribonuclease with antitumoral activity. Biochemistry 42: 11443-11450, 2003.

26. Juan G, Ardelt B, Li X, Mikulski SM, Shogen K, Ardelt W, Mittelman A and Darzynkiewicz Z: G1 arrest of U937 cells by onconase is associated with suppression of cyclin D3 expression, induction of p16INK4A, p21WAF1/CIP1 and p27KIP and decreased $\mathrm{pRb}$ phosphorylation. Leukemia 12: 1241-1248, 1998.
27. Rybak SM, Pearson JW, Fogler WE, Volker K, Spence SE, Newton DL, Mikulski SM, Ardelt W, Riggs CW, Kung HF, et al: Enhancement of vincristine cytotoxicity in drug-resistant cells by simultaneous treatment with onconase, an antitumor ribonuclease. J Natl Cancer Inst 88: 747-753, 1996.

28. Pavlakis N and Vogelzang NJ: Ranpirnase - an antitumour ribonuclease: Its potential role in malignant mesothelioma. Expert Opin Biol Ther 6: 391-399, 2006. 